\title{
Enhancement of urate solubility by connective tissue. II. Inhibition of sodium urate crystallisation by cation exchange
}

\author{
ELAINE PERRICONE AND KENNETH D. BRANDT \\ From the Rheumatology Division, Indiana University School of Medicine, Indianapolis, Indiana 46223, USA
}

SUMMARY The urate concentration of the supernatant was greater after supersaturated solutions of sodium urate were incubated in a suspension of CM-Sephadex C-25 than in one of Sephadex G-25. The supernatant urate concentration was greater when the CM-Sephadex had been equilibrated with potassium than with sodium. The results are analogous with those obtained in studies of urate solubility in proteoglycan solutions. They are consistent with the Donnan effect and the hypothesis that the glycosaminoglycans within the proteoglycan molecule function as cation exchangers which, when charged with potassium, exchange with the sodium of the urate molecule, leading to formation of highly soluble potassium urate.

Katz and Schubert (1970) have shown that proteoglycan solutions may enhance urate solubility, and have suggested that these molecules may play a role in preventing deposition of urate crystals in connective tissue in man. In recent studies aimed at interpreting that evidence in the light of recent knowledge of the macromolecular organisation of proteoglycans in tissue we found (Perricone and Brandt, 1978) that proteoglycan aggregates (that is, macromolecular complexes composed of several proteoglycan molecules non-covalently linked to a single molecular of hyaluronic acid) (Hascall and Heinegard, 1974) temporarily inhibited crystallisation of sodium urate from supersaturated solutions. Since this effect was considerably greater when potassium rather than sodium was the predominant cation in the solution, a question was raised concerning its significance in vivo, where sodium is the predominant extracellular cation. The exclusion of urate from the proteoglycan aggregates was consistent with the Donnan effect (Donnan, 1911), and we postulated that the glycosaminoglycans within the proteoglycan aggregates functioned as cation exchangers which, when charged with potassium, exchanged with the sodium of the urate molecule, leading to formation of highly soluble potassium urate.

Accepted for publication 24 November 1978

Correspondence to Kenneth D. Brandt, MD, Rheumatology Division, Indiana University School of Medicine, 1100 West Michigan Street, Indianapolis, Indiana 46223, USA.
To test this hypothesis we have herein examined the effect of the cation exchange resin CM Sephadex C-25 on the precipitation of urate from supersaturated solutions. We considered that the CM Sephadex bead, because of its high content of carboxyl groups, might affect urate solubility in a manner analogous to that of the proteoglycan aggregate. The results have been compared with those obtained with Sephadex G-25, whose pore size is the same as that of Sephadex C-25 but which lacks the carboxyl groups of the latter and is not an ion exchange resin.

\section{Materials and methods}

All reagents were of analytical grade with the exception of CM-Sephadex C-25 and Sephadex G-25 (medium) (Pharmacia Fine Chemicals; Piscataway, New Jersey). Microcrystalline sodium urate (NaU) was prepared from uric acid (Pfanstiehl Laboratories, Inc.; Waukegan, Illinois) according to the method of Seegmiller et al., (1962). Polarisation microscopy confirmed that the crystals were needle shaped.

Uric acid was determined by an enzymaticcolorimetric method (Boehringer Mannheim Corp.; Schaumberg, Illinois).

Solubility of Sodium Urate in the Presence of CM-Sephadex C-25 and Sephadex G-25. $100 \mathrm{mg}$ of CM-Sephadex C-25 or Sephadex G-25 was added to $1 \mathrm{ml}$ of either $0.021 \mathrm{M} \mathrm{K}_{2} \mathrm{HPO}_{4}-0.13 \mathrm{M} \mathrm{NaC1}$, pH 7.4 , or $0.021 \mathrm{M} \quad \mathrm{K}_{2} \mathrm{HPO}_{4}-0.13 \mathrm{M} \mathrm{KC1}$, 
pH 7.4 (hereafter designated as sodium buffer and potassium buffer, respectively), which had been sterilised by micropore filtration. The resin was equilibrated with the buffer by heating in a boiling water bath for 1 hour.

Supersaturated urate solutions were prepared by autoclaving an excess $(5.3 \mathrm{mg})$ of sodium urate crystals in $1 \mathrm{ml}$ of the sodium buffer. After cooling to $25^{\circ} \mathrm{C}$ the urate solutions were centrifuged and $1 \mathrm{ml}$ of the supernatant (urate concentration, $1.62 \mathrm{mg} / \mathrm{ml}$ ) and $50 \mu \mathrm{l}$ of a solution containing penicillin $(500 \mathrm{U})$, streptomycin $(50 \mu \mathrm{g})$, and Fungizone (125 pcg) were added to the resin suspension. The tubes were allowed to stand at $25^{\circ} \mathrm{C}$ for 16 hours, after which the samples were centrifuged for 20 minutes at 3000 r.p.m. The supernatants were removed by pipetting and, after an aliquot was taken for bacteriological culture the urate content was determined. Each experiment was performed in triplicate.

\section{Results and discussion}

The volume of the supernatant recovered after centrifugation of the tubes containing Sephadex G-25 was $1.6 \mathrm{ml}$ (Table 1). Based on the bed volume of the gel (4-6 ml/g) (Pharmacia Fine Chemicals, 1969) all of the buffer not recovered in the supernatant $(0.4 \mathrm{ml})$ could have been contained within the resin. This fraction (total solvent volume - supernatant volume) is hereafter designated $V_{i}$ (while $\mathrm{V}_{\mathbf{i}}$ conventionally denotes the inner volume of the gel, under the conditions of the present study $V_{i}$ will include also the small volume of solvent between the centrifuged resin beads). No urate crystals were observed within or between the beads after a portion of the pelleted resin was frozen, sectioned $(4 \mu \mathrm{m})$ with a cryostat, mounted by air drying on microscope slides, and viewed by polarisation microscopy.
Based on the measured urate concentrations $(0.74-0.76 \mathrm{mg} / \mathrm{ml})$ and the volume $(1.6 \mathrm{ml})$ of the Sephadex G-25 supernatants their urate contents represented $73 \%$ and $75 \%$, respectively, of the total urate when the resin had been equilibrated with the sodium and potassium buffers (Table 1). On $\overrightarrow{\widetilde{\sigma}}$ the assumption that the urate concentration of the Sehadex G-25 $V_{i}$ was identical to that of the super-es natant (Fig. 1) it may be calculated that $18 \%$ of the $\vec{\circ}$ total urate content was contained in $\mathrm{V}_{\mathrm{i}}$. A smallproportion $(7-9 \%)$ of the total urate cannot be accounted for in the supernatant or in $V_{i}$, and waso

\section{$\mathrm{Na}^{+} \mathbf{U}^{-}$}

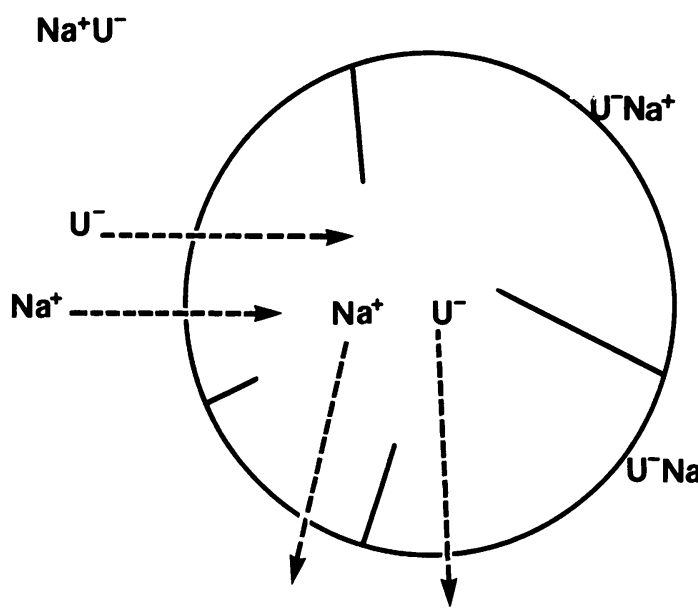

Fig. 1 Distribution of sodium urate $\left(\mathrm{Na}^{+} \mathrm{U}^{-}\right)$in a suspension of Sephadex $G-25$ resin. Sodium and urate ions in solution within the resin bead are in equilibrium with sodium and urate ions in solution outside the bead. Adsorption of 2 urate ions on the surface of the resin is depicted on the right hand side of the bead

Table 1 Effects of sodium and potassium forms of Sephadex $G-25$ and $C M$-Sephadex $C-25$ on urate concentrations in $0.021 \mathrm{M} \mathrm{K}_{2} \mathrm{HPO}_{4}-0.13 \mathrm{M} \mathrm{NaCl}, \mathrm{pH} 7.4$

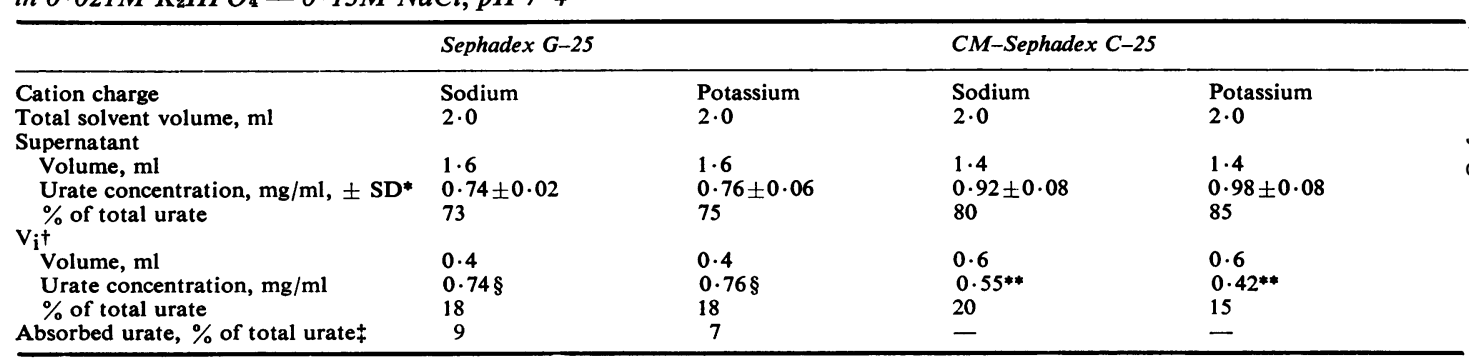

* Mean, triplicate samples. + Total solvent volume-supernatant volume. $¥$ Total urate content-(supernatant urate content $+\mathrm{V}_{\mathrm{i}}$ urate content)/total urate content $\times 100$. $\S$ Assumed, from urate concentration of supernatant. ** Total urate content-supernatant urate content $/ \sigma$ volume of $\mathbf{V}_{\mathbf{i}}$. 
presumably adsorbed on to the resin (Fig. 1). This is not unreasonable, since another purine, xanthine, is adsorbed by Sephadex G-25 to a similar extent (Gelotte, 1960).

While $80 \%$ of the total solvent volume was recovered in the supernatant of tubes which contained Sephadex G-25, the CM-Sephadex C-25 supernatant accounted for only $70 \%$ of the solvent volume. Since the bed volume of the latter resin is 6-10 ml/g (Pharmacia Fine Chemicals, 1970), most of the solvent not in the supernatant could be accounted for in the CM-Sephadex $\mathrm{V}_{\mathrm{i}}$. Polarisation microscopy of pellets of the CM-Sehadex, prepared as described above, showed no crystals within or around the resin.

When the resins were equilibrated with potassium buffer the urate concentration of the CM-Sephadex supernatant was greater than that of the Sephadex G-25 supernatant $(0.98$ and $0.76 \mathrm{mg} / \mathrm{ml}$, respectively; $\mathbf{P}<0.025)$. Similarly, after equilibration of the resins with sodium buffer the urate concentration of the CM-Sephadex supernatant was greater than that of the Sephadex G-25 supernatant $(0.92$ and $0.74 \mathrm{mg} / \mathrm{ml}$, respectively; $\mathbf{P}<0.025$ ). Presumably the fixed negatively charged carboxyl groups on the CM-Sephadex repelled urate ions seeking to enter the interstices of the beads, leading to a greater urate concentration in the supernatant

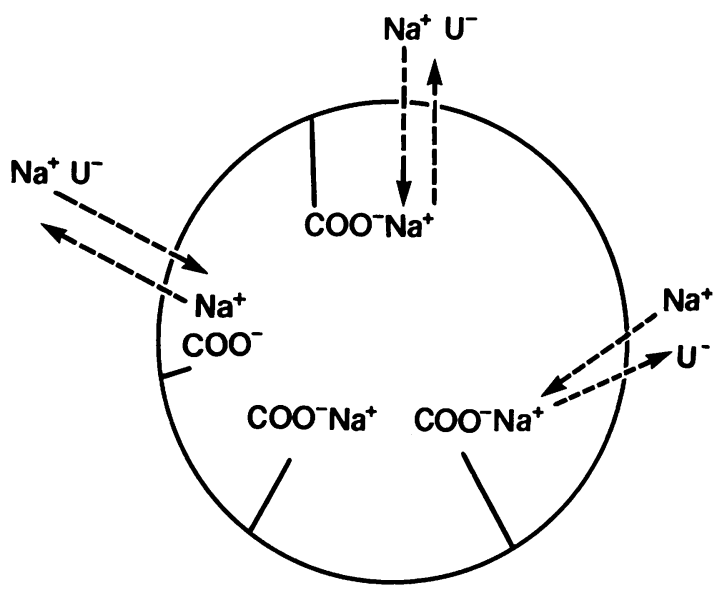

\section{$\mathrm{Na}^{+} \mathrm{U}^{-}$}

Fig. 2 Distribution of sodium urate $\left(\mathrm{Na}^{+} \mathrm{U}^{-}\right)$in a suspension of Sephadex CM-25 resin in the sodium form. Sodium ions within the beads equilibrate freely with those in solution outside the beads. The negatively charged urate ions, however, are repelled by the fixed carboxyl groups on the resin and tend to remain outside, creating a concentration gradient for urate. than in $V_{i}$ (Fig. 2 and 3). Based on the total urate and the urate concentration of the supernatant, and on the assumption of no adsorption of urate on to the resin, the urate concentration in the CM-Sephadex $\mathrm{V}_{\mathrm{i}}$ can be calculated to have been $0.42-0.55 \mathrm{mg} / \mathrm{ml}$ (Table 1).

The somewhat greater urate concentration noted in the supernatant when CM-Sephadex was equilibrated with the potassium buffer than when it was equilibrated with the sodium buffer may be explained as follows. Under these conditions potassium ions associated with the resin would have exchanged with sodium ions from the sodium urate solution (Fig. 3a and $b$ ). As they were replaced by sodium the potassium ions, following a concentration gradient, would have flowed out of the beads where urate ions, repelled by the fixed carboxyl groups on the resin, would have tended to reside. Thus conditions would have favoured the formation of potassium urate outside of the beads. The much greater solubility of potassium urate than sodium urate (Perricone and Brandt, 1978) accounts for the greater urate concentration observed in the supernatant when CMSephadex had been charged with potassium than when it had been charged with sodium.

The above results are similar to those obtained when proteoglycan aggregates were employed instead of CM-Sephadex. The observed ion exclusion phenomena can be explained by the Donnan effect (Donnan, 1911) and are consistent with the hypothesis (Perricone and Brandt, 1978) that the greater enhancement of urate solubility by the potassium salts of proteoglycans than by their sodium salts is due to ion exchange on the

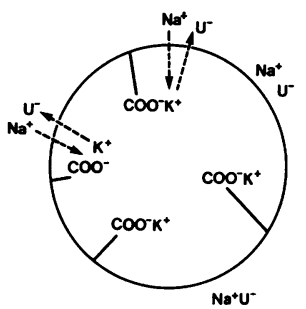

(a)

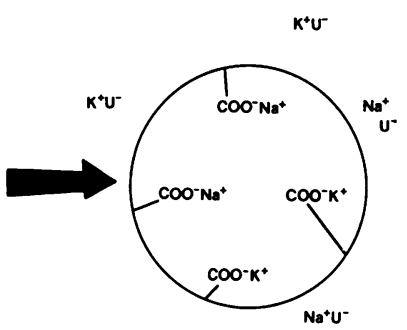

(b)
Fig. 3 Distribution of sodium urate $\left(\mathrm{Na}^{+} \mathrm{U}^{-}\right)$in a suspension of Sephadex $C M-25$ resin in the potassium form. (a) Potassium ions within the beads exchange freely with sodium ions in the solution outside. The negatively charged urate ions, however, are repelled by the fixed carboxyl on the resin and tend to remain outside, creating a concentration gradient for urate. (b) Equilibration of the resin thus leads to formation of highly soluble potassium urate outside the bead 


\section{Perricone, Brandt}

glycosaminoglycan chains of the proteoglycans, and the greater solubility of potassium urate than of sodium urate.

We are grateful to Dr Larry Steinrauf, of the Biochemistry Department, Indiana University School of Medicine, for his helpful comments, and to Lorna Bowman for her excellent secretarial support.

This work was supported in part by grants from the National Institutes of Arthritis, Metabolic and Digestive Diseases (AM 20582), the Arthritis Foundation, the Grace M. Showalter Trust, and the Burroughs Wellcome Co.

\section{References}

Donnan, F. G. (1911). The theory of membrane equilibrium in the presence of a non-dialyzable electrolyte. Zeitschrift für Elektrochemie und angewandte physikalische Chemie, 17, 572.
Gelotte, B. (1960). Studies on gel filtration. Sorption proper ties of the bed material Sephadex. Journal of Chromato $\Rightarrow$ graphy, 3, 330-342.

Hascall, V. C., and Heinegard, D. (1974). Aggregation of cartilage proteoglycans. I. The role of hyaluronic acid Journal of Biological Chemistry, 249, 4232-4241.

Katz, W. A., and Schubert, M. (1970). The interaction of monosodium urate with connective tissue components $\mathbb{D}^{\mathbb{D}}$ Journal of Clinical Investigation, 49, 1783-1789.

Perricone, E., and Brandt, K. (1978). Enhancement of urate solubility by connective tissue. I. Effect of proteoglycan aggregates and buffer cation. Arthritis and Rheumatism, 21; 453-460.

Pharmacia Fine Chemicals (1970). Ion Exchangers, an Outstanding Aid in Biochemistry. Uppsala.

Pharmacia Fine Chemicals (1969). Sephadex-Gel Filtration in Theory and Practice. Uppsala.

Seegmiller, J. E., Howell, R. R., and Malawista, S. E. (1962) The inflammatory reaction to sodium urate: its possible relationship to the genesis of acute gouty arthritis. JournaD of the American Medical Association, 180, 469-475. 